\title{
Predictability Modulates Human Brain Response to Reward
}

\author{
Gregory S. Berns, ${ }^{1}$ Samuel M. McClure, ${ }^{2}$ Giuseppe Pagnoni, ${ }^{1}$ and P. Read Montague ${ }^{2}$ \\ ${ }^{1}$ Department of Psychiatry and Behavioral Sciences, Emory University School of Medicine, Atlanta, Georgia 30322, and \\ ${ }^{2}$ Center for Theoretical Neuroscience, Division of Neuroscience, Baylor College of Medicine, Houston, Texas 77030
}

\begin{abstract}
Certain classes of stimuli, such as food and drugs, are highly effective in activating reward regions. We show in humans that activity in these regions can be modulated by the predictability of the sequenced delivery of two mildly pleasurable stimuli, orally delivered fruit juice and water. Using functional magnetic resonance imaging, the activity for rewarding stimuli in both the nucleus accumbens and medial orbitofrontal cortex was greatest when the stimuli were unpredictable. Moreover, the sub-
\end{abstract}

jects' stated preference for either juice or water was not directly correlated with activity in reward regions but instead was correlated with activity in sensorimotor cortex. For pleasurable stimuli, these findings suggest that predictability modulates the response of human reward regions, and subjective preference can be dissociated from this response.

Key words: reward; dopamine; fMRI; reinforcement; neural network; nucleus accumbens; striatum
The pursuit of natural rewards such as food, drink, and sex is a major external influence on human behavior. Nevertheless, the issue of how rewards affect human behavior remains primarily unresolved. There are many factors that contribute to this gap in our knowledge; however, one roadblock has been the difficulty of defining and measuring isolated effects of rewards on human behavior or brain activation. In animals, reward is defined as an operational concept: a stimulus is deemed rewarding if it positively reinforces a behavior (Hull, 1943; Rescorla and Wagner, 1972; Robbins and Everitt, 1996), that is, reliably increases the likelihood of the behavior. The same concept applies to humans; however, humans have the ability to exert all sorts of executive control over their actions, and so behavioral assays alone are an incomplete way to probe reward processing. Similarly, explicit reports of likes and dislikes, i.e., preferences, are confounded by an individual's subjective perception of what they like and what they choose to report. To overcome these experimental difficulties, one would like to monitor concurrently behavioral output, subjective preference, and brain response during a well defined task. Taking such an approach, we report here that activity in human reward regions is more closely correlated with the predictability of a sequence of pleasurable stimuli than with explicitly stated preferences.

In humans, activation of reward areas can be visualized with functional magnetic resonance imaging (fMRI) after administration of drugs, such as cocaine (Breiter et al., 1997); however, such infusions may not be representative of normal reward processing because of both direct and indirect pharmacological effects of cocaine. Furthermore, drugs such as cocaine may act on different

Received Nov. 11, 2000; revised Jan. 17, 2001; accepted Jan. 26, 2001.

This work was supported by National Institute on Drug Abuse Grants K08 DA00367 (to G.S.B.) and RO1 DA11723 (to P.R.M.), the National Alliance for Research in Schizophrenia and Depression (G.S.B.), and the Kane Family Foundation (P.R.M.). We thank H. Mao, R. King, and M. Martin for their assistance with data collection.

Correspondence may be addressed to either Gregory S. Berns, Department of Psychiatry and Behavioral Sciences, Emory University School of Medicine, 1639 Pierce Drive, Suite 4000, Atlanta, GA 30322, E-mail: gberns@emory.edu, or P. Read Montague, Division of Neuroscience, Baylor College of Medicine, 1 Baylor Plaza, Houston, TX 77030, E-mail: read@bcm.tmc.edu.

Copyright (C) 2001 Society for Neuroscience 0270-6474/01/212793-06\$15.00/0 parts of the reward system than so-called natural rewards such as food and water (Bradberry et al., 2000; Carelli et al., 2000). Conditioned rewards, e.g., money, may also act on different parts of the reward system (Thut et al., 1997; Elliott et al., 2000; Knutson et al., 2000) and may not be an appropriate probe of primary reward circuits in humans. An alternative approach is suggested by experiments that demonstrate that the predictability of a primarily rewarding stimulus is a critical parameter for activation of reward pathways (Schultz et al., 1992, 1997; Schultz, 1998; Garris et al., 1999). Physiological recordings in nonhuman primates have demonstrated that neurons in regions such as the ventral tegmental area (VTA), nucleus accumbens, and ventral striatum respond in an adaptive manner to rewarding stimuli such as fruit juice or water (Shidara et al., 1998). Thus, the predictability of a sequence of stimuli may itself recruit reward-related neural structures in a manner detectable with fMRI. Moreover, theoretical models of dopamine release suggest that unpredictable rewards should elicit greater activity in these regions (Schultz et al., 1997). We sought to test this hypothesis by using fMRI to measure the effect of predictability on human brain responses to sequences of punctate, pleasurable stimuli.

\section{MATERIALS AND METHODS}

Subjects. Twenty-five normal adults underwent fMRI scanning while being administered small amounts of either oral fruit juice or water. The subjects ranged in age from 18 to 43 , and all subjects gave informed consent for a protocol approved by the Emory University Human Investigations Committee.

Experimental task. While in the scanner, subjects received small amounts of orally delivered fruit juice and water in either a predictable or unpredictable manner. We chose a sequenced delivery of fruit juice and water for three reasons: (1) humans find both juice and water to be subjectively pleasurable; (2) both stimuli are routinely used as reinforcing stimuli while training nonhuman primates on behavioral tasks; and (3) midbrain dopaminergic neurons, and presumably the neurons to which they project, show phasic changes in firing rate as a function of the temporal predictability of sequential stimuli (Schultz et al., 1992). Participants received both the juice and the water in either a predictable or unpredictable manner in two scanning runs (Fig. 1). During the predictable run, juice and water boluses were alternated at a fixed interval of 10 sec. During the unpredictable run, the order of juice and water was randomized, and the stimulus interval was also randomized by sampling a Poisson interval distribution with a mean of $10 \mathrm{sec}$. Each run lasted 5 


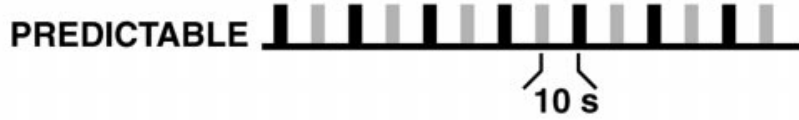

\section{UNPREDICTABLE}

Figure 1. Design of the fMRI experiment. A $2 \times 2$ factorial design was used, with factors of preference (juice or water) and predictability (predictable or unpredictable). Subjects received $0.8 \mathrm{ml}$ boluses of juice and water in either a predictable or unpredictable sequence. Using eventrelated fMRI, brain activation was analyzed in terms of preference and predictability, as well as the interaction between them.

min, and the order of the two runs (predictable or unpredictable) was randomized across subjects. Because the time to adapt to either predictability or unpredictability was unknown and because frequent switching of conditions might cause an interaction with each other, i.e., the "predictability of predictability," we chose to separate the conditions across scan runs rather than use smaller condition blocks within scan runs. Because all aspects of the experiment hinged on manipulating predictability, we chose to not repeat conditions within subjects and instead focused on studying a larger number of subjects.

Subjects received $0.8 \mathrm{ml}$ oral boluses of both fruit juice and water via two plastic tubes. A mouthpiece held the ends of the tubing in place over the tongue, with the fruit juice inf used from the left side of the mouthpiece and the water from the right. The tubes were $\sim 10 \mathrm{~m}$ long and were connected to a computer-controlled dual-syringe pump (Harvard Apparatus, Holliston, MA) outside the scanner room. Subjects did not perform any other task during scanning and were instructed to simply swallow the fluid each time it was administered. After the scan session, subjects were debriefed for their fluid preference.

Acquisition of MRI data. Scanning was performed on a 1.5 Tesla Philips NT scanner. After acquisition of a high-resolution T1-weighted anatom- ical scan, subjects underwent two whole-brain functional runs of 150 scans each (echo-planar imaging, gradient recalled echo; repetition time, $2000 \mathrm{msec}$; echo time, $40 \mathrm{msec}$; flip angle, 90 $64 \times 64$ matrix, $245 \mathrm{~mm}$ axial slices acquired parallel to anteroposterior commissural line) for measurement of the blood oxygenation level-dependent (BOLD) effect (Kwong et al., 1992; Ogawa et al., 1992). Head movement was minimized by padding and restraints.

Analysis. The data were analyzed using Statistical Parametric Mapping (SPM99; Wellcome Department of Cognitive Neurology, London, UK) (Friston et al., 1995b). Motion correction to the first functional scan was performed within subjects using a six-parameter rigid-body transformation. Because swallowing unavoidably causes significant head movement, the motion-correction parameters were also used to determine whether head motion differed significantly between the conditions. The mean of the motion-corrected images was then coregistered to the individual's 24-slice structural MRI using a 12-parameter affine transformation. The images were then spatially normalized to the Montreal Neurological Institute (MNI) template (Talairach and Tournoux, 1988) by applying a 12-parameter affine transformation, followed by a nonlinear warping using basis functions (Ashburner and Friston, 1999). Images were subsequently smoothed with an $8 \mathrm{~mm}$ isotropic Gaussian kernel and bandpass filtered in the temporal domain. A random-effects, event-related, statistical analysis was performed with SPM99 (Friston et al., 1995a, 1999). The experiment was analyzed as a $2 \times 2$ factorial design. First, a separate general linear model (GLM) was specified for each subject, with four conditions representing the four possible event types: predictablepreferred fluid, predictable-nonpreferred fluid, unpredictable-preferred fluid, and unpredictable-nonpreferred fluid. Four vectors of delta functions with times corresponding to each event were created for each of the four conditions. These were convolved with a generic hemodynamic response function and entered into a four-column design matrix. The mean of each scan run was removed on a voxelwise basis. We calculated three two-sided contrast images that corresponded to the main effects of preference [contrast vector (1-11-1)], predictability [contrast vector (11-1-1)], and the interaction term [contrast vector (1-1-11)]. The interaction describes how predictability modulates the effect of preference. These individual contrast images were entered into a second-level analysis, using a separate one-sample $t$ test $(\mathrm{df}=24)$ for each side of each term in the GLM (a total of six contrasts). We thresholded these summary statistical maps at $p<0.001$ (uncorrected for multiple com-

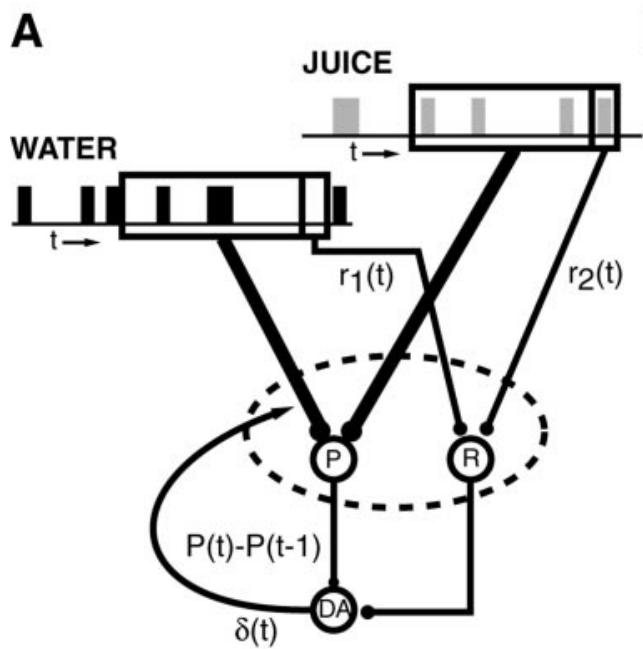

B
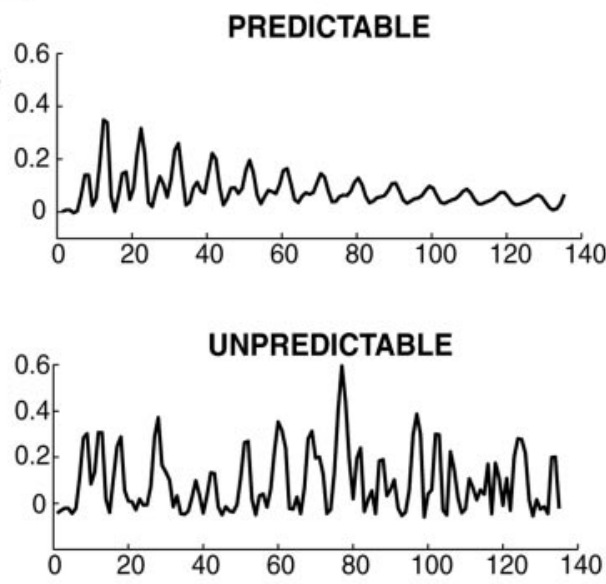

Figure 2. Neural network model of the experiment and the brain regions associated with information processing. $A$, Diagram indicates our hypothesis for how the sequence of stimuli could influence dopaminergic output. In this hypothesis, we have indicated that changes in dopaminergic output could influence target neural structures in a manner detectable in a fMRI BOLD measurement. The juice and water are shown to have both sensory (projection from finite time window box) and reward (the $r$ pathways) representations in their influence on dopaminergic activity. To generate an expected hemodynamic response from this hypothesis, we made a finite time window (small boxes for juice and water), which determined the value of the immediate reward $r(t)$ ( 1 if juice occurred, 0.5 if water occurred, and 0 if no stimulus occurred). This maneuver arbitrarily set juice to twice the value of water. This is not important for the main expectation generated by the model. $B$, Predicted dopamine effect for predictable and unpredictable sequences of juice and water delivery. Horizontal axis is scan number. Vertical axis is the expected hemodynamic response predicted by a temporal difference model. The scale on the vertical axis is arbitrary. The important point to note is that the predictable run progresses to 0 , whereas the unpredictable run remains high-amplitude throughout. The traces were generated by convolving a hemodynamic response kernel with the output of a temporal difference model. This suggested that the average BOLD response would be greater when the stimuli were unpredictable. 


\begin{tabular}{|c|c|c|c|}
\hline Brain region & $\begin{array}{l}\text { MNI coordinates } \\
(x, y, z)\end{array}$ & $\begin{array}{l}\text { Peak } t \\
\text { value }\end{array}$ & Cluster size \\
\hline \multicolumn{4}{|l|}{ Preferred $>$ nonpreferred } \\
\hline L somatosensory cortex & $-60,-12,16$ & 4.19 & $17(p<0.01)$ \\
\hline \multicolumn{4}{|l|}{ None } \\
\hline \multicolumn{4}{|l|}{ Unpredictable $>$ predictable } \\
\hline $\mathrm{R}$ medial orbitofrontal cortex including & $20,36,-12$ & 7.31 & 77 \\
\hline Bilateral nucleus accumbens & $0,16,-8$ & 7.17 & \\
\hline Bilateral paracentral lobule & $12,-20,64$ & 6.35 & 239 \\
\hline $\mathrm{L}$ mediodorsal nucleus of thalamus & $-4,-20,4$ & 5.86 & 12 \\
\hline \multirow[t]{2}{*}{$\mathrm{R}$ cerebellum } & $44,-68,-24$ & 5.85 & 12 \\
\hline & $16,-76,-28$ & 4.86 & 26 \\
\hline \multicolumn{4}{|l|}{ Predictable $>$ unpredictable } \\
\hline $\mathrm{R}$ superior temporal gyrus & $56,-40,16$ & 5.16 & 98 \\
\hline $\mathrm{L}$ precentral gyrus & $-48,4,36$ & 4.28 & 14 \\
\hline R lateral orbitofrontal gyrus & $32,16,-16$ & 4.22 & 13 \\
\hline \multicolumn{4}{|c|}{ (Preferred-nonpreferred $) \times($ unpredictable-predictable $)$} \\
\hline L superior temporal gyrus & $-48,-4,-16$ & 3.15 & $5(p<0.01)$ \\
\hline \multicolumn{4}{|c|}{$($ Preferred-nonpreferred $) \times($ predictable-unpredictable $)$} \\
\hline $\mathrm{R}$ insula & $44,20,12$ & 3.61 & $17(p<0.01)$ \\
\hline $\mathrm{L}$ posterior cingulate gyrus & $-12,-40,8$ & 3.59 & $46(p<0.01)$ \\
\hline $\mathrm{R}$ cerebellum & $28,-52,-32$ & 3.56 & $13(p<0.01)$ \\
\hline
\end{tabular}

L, Left; R, right.

parisons). These maps were overlaid on a high-resolution structural image in MNI orientation.

Theoretical model. As a tool for both designing and interpreting the fMRI experiment, we used an existing neural network model of dopamine release to simulate the brain response to different temporal patterns of rewarding stimuli (Fig. 2). This model was based on the method of temporal differences (TD), which postulates that a synaptically reinforcing substance, e.g., dopamine, is released in response to errors in reward prediction (Schultz et al., 1997). This model has been used in a wide variety of applications, including complex learning tasks such as backgammon (Sutton, 1988; Tesauro and Sejnowski, 1989), as well as successfully predicting the activity of dopamine neurons in numerous conditioning paradigms (Houk et al., 1995; Montague et al., 1995) and motor sequencing tasks (Berns and Sejnowski, 1998).

Briefly, TD learning depends on two primary assumptions. First, short-term adaptation in a given neural circuit occurs with the goal of predicting a discounted sum of all future rewards. The definition of a reward depends on the context in which it is received. If a putative reward increases the occurrence of a particular behavior, then it is deemed a positive reinforcer. Depending on the internal state of the animal, the same reward may not reinforce a behavior, e.g., when the animal is satiated. In the context of an fMRI experiment, which is generally unnatural, a familiar appetitive substance such as water or fruit juice is subjectively experienced as pleasant and therefore rewarding. Second, reward predictions depend only on the current representation of a stimulus set. The stimulus representation is somewhat arbitrary in the model, and it includes some representation backward through time, i.e., a stimulus trace. For substances such as water or fruit juice, there exist both sensory dimensions (e.g., temperature and tactile sensation on the tongue) and the actual reward, which is subjectively experienced as pleasure. Therefore, it is reasonable to consider the tactile dimensions of fluid delivery as both neutral and distinct from the rewarding dimension. Similarly, these distinct dimensions are presumed to be processed by different brain circuits, which can be imaged with fMRI. To map the model output onto a dimension analogous to the measurement obtained with fMRI, we summed the outputs of both the neutral and rewarding pathways, which we assumed converged in the ventral striatum and nucleus accumbens. We acknowledge that there is no direct evidence for this, and depending on the specific receptor, dopamine can have variable effects on neuronal activity. The exact experimental design was input to the model, which was simulated with Matlab 5.3 (MathWorks, Natick, MA). The outputs corresponding to both the putative dopamine neurons and their projection sites were calculated for the predictable and unpredictable runs (Fig. 2).

We should be careful to point out to readers that our use of the temporal difference model to explain our design and subsequent interpretation (below) is based on its previous success in describing changes in spike output in dopaminergic neurons in primates undergoing related behavioral tasks. There are other plausible computational descriptions that could also suffice.

\section{RESULTS}

After the scans, subjects were queried about their preference for the two stimuli. Eighteen of 25 subjects $(72 \%)$ preferred juice, and the remainder preferred water. Most subjects had a distinct preference for one or the other, although we did not ask them to quantify this. Although there was significant head motion during the scans, all of the translations and rotations around each stimulus were generally small and were not significantly different between any of the conditions. For example, the mean $\pm \mathrm{SD}$ translation associated with each stimulus was $0.041 \pm 0.069 \mathrm{~mm}$ in the predictable condition and $0.044 \pm 0.069 \mathrm{~mm}$ in the unpredictable condition (paired $t$ test; $p=0.853$ ).

The brain response to the preferred fluid displayed surprisingly little differential activity relative to the nonpreferred fluid (Table 1). We did not observe any significant activity difference in classical reward regions such as the nucleus accumbens, hippocampus, or medial prefrontal cortex. The primary activity change for preferred $>$ nonpreferred occurred in the somatosensory cortex in an area near the mouth and tongue region $(t=4.19$, MNI coordinates, $-60,-12,16)$.

The main effect of predictability was substantially greater than the main effect of preference (Fig. 3). For the unpredictable run relative to the predictable run, bilateral activation was observed 


\section{A Unpredictable - Predictable}

Figure 3. The main effect of predictability showed that reward-related regions had a greater BOLD response to the unpredictable stimuli. $A$, Planes centered at $(0,4,-4)$ show that bilateral nucleus accumbens/ventral striatum $(N A C)$ and bilateral superior parietal cortex were more active in the predictable condition. $B$, A small region in the right superior temporal gyrus was relatively more activated by the predictable stimuli. Significance was thresholded at $p<0.001$ and an extent $>10$ contiguous voxels.
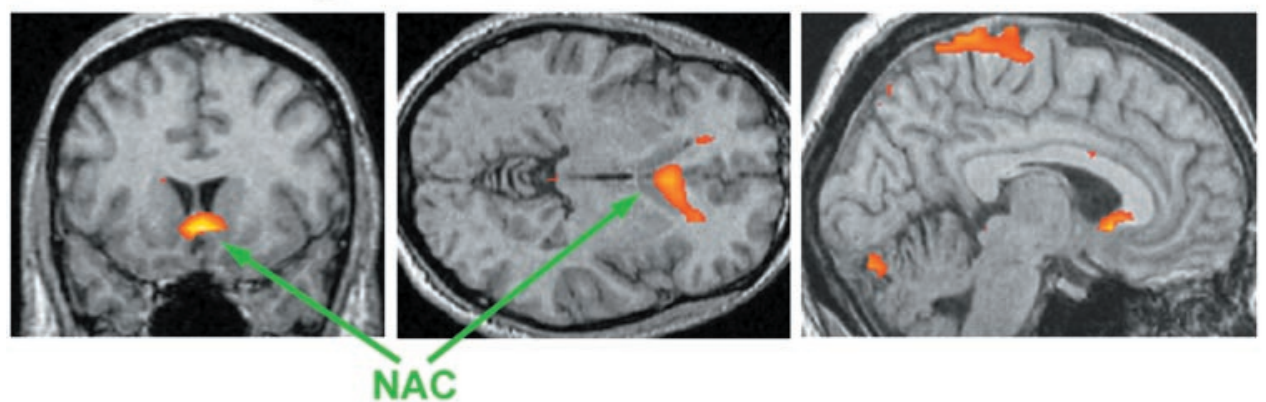

\section{B Predictable - Unpredictable}

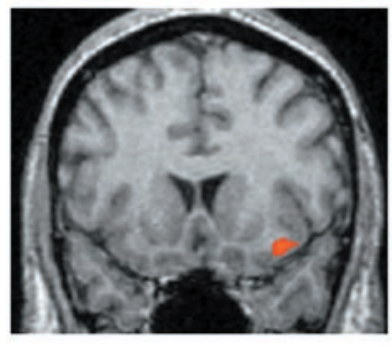

$\mathrm{T}_{24}$

0
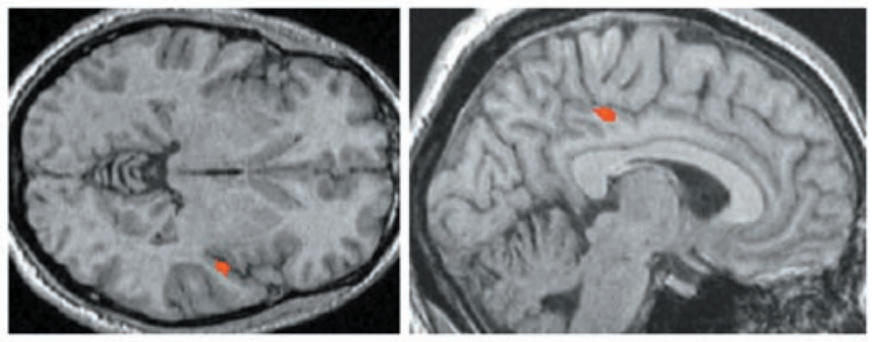

7 in a large expanse of medial orbitofrontal cortex that included the nucleus accumbens (Table 1). Additional areas of activation included a large area of parietal cortex bilaterally and paracentrally and small focal activations in both the left mediodorsal nucleus of the thalamus and right cerebellum. Because none of these regions overlapped with the main effect of preference, they were maximally activated by unpredictable stimuli, regardless of preference. For the predictable run relative to the unpredictable run, an area of the right superior temporal gyrus was activated, as well as focal activations in the left precentral gyrus and right lateral orbitofrontal cortex.

The interaction between preference and predictability identified areas in which one effect modulated the other independently of both main effects. The right insula, left posterior cingulate, and right cerebellum displayed a significant interaction for the contrast (preferred-nonpreferred) $\times$ (predictable-unpredictable). The opposite contrast, (preferred-nonpreferred) $\times$ (unpredictable-predictable), did not reveal any activations significant at the $p<0.001$ level; however, a small region in the left superior temporal gyrus (MNI coordinates, $-48,-4,-16$ ) was significant at the $p<0.01$ level $(t=3.15)$.

The computer simulation suggested that unpredictable rewards should evoke more dopamine release than predictable ones (Fig. $2 B$ ). When the rewards are predictable, each stimulus perfectly predicts the subsequent one, and the error signal, which is presumed to be mediated by dopamine, gradually decreases. When the rewards are unpredictable, there is no opportunity for the system to learn, and the response to each stimulus is greater.

\section{DISCUSSION}

Our results demonstrated an interesting separation in the brain response to predictability and to subjective reports of preference. The brain response to preference was exclusively cortical, but the response to predictability showed specific activation of reward systems also known to be the target of midbrain dopaminergic neurons. If we presume that activation of these reward areas is pleasurable to humans, then this finding suggests that the subjective report of preference may be dissociated from neural circuits known to be powerful determinants of conditioned behaviors.

Both the water and the fruit juice caused significant activations throughout the brain, and although some of this response was attributable to the motoric aspects of the task, specific subsets of these regions were decomposed into dimensions of preference and predictability. The effect of preference was restricted to cortical regions associated with sensory processing, and the preferred stimulus resulted in greater activation in these regions. These regions lie near sensorimotor cortex known to be activated during tongue movements (Corfield et al., 1999) and swallowing (Hamdy et al., 1999). In previous work on the brain response to tongue movement, there was substantial activation of the cerebellum, a finding notably absent in the main effect of preference. The differential brain response, i.e., preferred-nonpreferred, removes common regions of activation; therefore, the absence of cerebellar activation suggests that differential tongue movements were unlikely to be the cause of the pattern of cortical activation for subjective preference. The fact that a somatosensory region was correlated with stated preference was suggestive that some differential neural processing occurred for the two stimuli. It was surprising that this was manifest in a primary sensory processing area and not in classical reward areas. Although subjects were forced to designate one substance over the other as their preference, both fluids were chosen purposely to be pleasurable, in contradistinction to one being aversive. Because both fluids were generally pleasurable, the effect of preference might not have been strong enough to result in a significant activity difference in reward regions. This would be consistent with findings that midbrain dopamine neurons are preferentially activated by appetitive 
rather than aversive stimuli (Mirenowicz and Schultz, 1996). Nevertheless, our findings suggest a system differentiation of subjective preference from simple reward, which supports previous hypotheses that "wanting" is not the same as "liking" (Robinson and Berridge, 1993).

Unlike the effect of preference, unpredictability correlated as a significant main effect with activity in the nucleus accumbens, thalamus, and medial orbitofrontal cortex, whereas predictability was correlated predominately with activity in the right superior temporal gyrus. The former regions correspond closely with known dopamine projection sites (Koob, 1992; Cooper et al., 1996). It was somewhat surprising that unpredictability, and not preference, was correlated with activity in these reward areas. If increased activity in these regions was associated with pleasure, then one might conclude that unpredictable rewards were more pleasurable than predictable ones. However, most of the subjects did not discern any difference between the predictable and unpredictable conditions. If the unpredictable rewards were more pleasurable than predictable ones, or vice versa, then this must be occurring at a subconscious level. An alternative explanation presumes that dopamine is released in increased amounts to unexpected rewards (Montague et al., 1996; Schultz et al., 1997; Schultz, 1998). Dopamine can decrease neuronal excitability (Cooper et al., 1996) and may also directly constrict the microvasculature (Krimer et al., 1998), but increased accumbens activity has also been associated with the subjective pleasure of cocaine (Breiter et al., 1997). These findings suggest that our observed increase in activation with unpredictability could be related to increased dopamine release, either because the accumbens projects to the VTA or because it receives a projection from the VTA, either of which would be consistent with the model results. This interpretation should be tempered by two important facts: (1) the mechanisms that would couple increased dopaminergic transmission to changes in the BOLD signal are unknown, and (2) we have no independent measure of dopaminergic transmission, only changes in the BOLD response. The possibility that we are observing indirectly changes in dopaminergic activity is exciting but cannot be decided unequivocally in an fMRI experiment. It is, however, consistent with previous findings using positron emission tomography that dopamine is released into the ventral striatum under conditions of monetary incentive (Koepp et al., 1998). Coupled with the amplifying effect of unpredictability, it is also consistent with hypothesized effects of dopamine on neuronal "gain" (Cohen and Servan-Schreiber, 1992), with the end result that some regions will increase and others will decrease.

The specific regions activated relatively by unpredictability corresponded to brain regions associated with appetitive functions. In addition to the nucleus accumbens, the medial orbitofrontal cortex showed a main effect for unpredictability. This region has been shown in primates to integrate both the rewarding and neutral aspects of taste sensations and is thought to reflect primarily the motivational values of these stimuli (Rolls, 2000). This region also contains neurons that discriminate relative preference for reward (Tremblay and Schultz, 1999). The orbitofrontal cortex is typically difficult to image with fMRI because of the susceptibility artifact from the nasal sinuses (Ojemann et al., 1997). However, the region that we identified is generally superior and caudal to the usual artifact location. This region has previously been found responsive to pleasant tastes (Francis et al., 1999). A second region, in the superior parietal lobe, was probably not related to the rewarding aspects of the task but rather the result of changes in attention. This region has previously been implicated in visuospatial attention, especially during expectation violations (Nobre et al., 1999). Another region, in the left temporal cortex, showed a borderline significant modulation by unpredictability. In recent fMRI experiments, the left temporal lobe has been associated with processing the predictability of sequential stimuli (Bischoff-Grethe et al., 2000). Here, we extend these previous findings from neutral stimuli to pleasurable stimuli, suggesting that this region may perform a generic monitoring of predictability independently of stimulus valence.

The brain regions that we identified as responding to unpredictability in either a direct or modulatory manner have been implicated in a number of experiments on financial reward. Money can be rewarding to humans, but it is reinforcing only because it has acquired these properties through complex conditioning. Similar to the finding that cocaine acts on different neurons than natural reinforcers (Carelli et al., 2000), it is possible that conditioned reinforcers, such as money, act on different neural systems than natural reinforcers such as food and water. Activity in both the ventral striatum and midbrain have been correlated with absolute levels of financial reward (Thut et al., 1997; Delgado et al., 2000; Elliott et al., 2000; Knutson et al., 2000), a finding notably absent in our results. As noted previously, both the juice and water were mildly pleasurable, and so there may not have been a substantial difference in absolute reward, although we assumed a slight difference in the theoretical model. Also, we did not use any aversive stimuli or anything that could be construed as a negative reward, which may also account for this difference. Interestingly, the regions we identified as being either directly affected or amplified by unpredictability corresponded to the regions found previously to be sensitive to the context dependence of the financial reward (Rogers et al., 1999; Elliott et al., 2000). In particular, both the subgenual cingulate and medial thalamus were correlated with unpredictability in our study and were found to be context-dependent by Elliott et al. (2000).

Because predictability modulated the effect of preference, it is important to distinguish the potential sources of prediction. In a classical conditioning experiment, a neutral stimulus precedes the reward. After training, the previously neutral stimulus becomes the predictor, or conditioned stimulus. Because there are comparatively few data on the use of oral stimuli in fMRI, we chose to simplify the experiment and control for the motoric aspects of the task by using two different oral stimuli, water and fruit juice. Thus, the source of prediction in our experiment necessarily came from the sequence of stimuli themselves. In some ways, this is simpler than introducing another stimulus modality, such as a visual cue, but because both stimuli were rewarding, we cannot make any conclusions regarding the process of conditioning. Both the theoretical model (Schultz et al., 1997) and neurophysiological data (Schultz et al., 1992, 1993) suggest that reward predictions are computed during the interval preceding reward delivery. Because we do not know the time scale over which such predictions are computed, we chose to analyze the experiment as simply two conditions, predictable and unpredictable. By maintaining a psychologically reasonable interval between stimuli, $10 \mathrm{sec}$, there was insufficient time to resolve differences in interstimulus processing. Presumably such processing does occur, and this could be resolved with a differently designed experiment.

In summary, activity in human reward regions can be modulated by the temporal predictability of primary rewards such as water and juice. These results provide important support for computational models that postulate that errors in reward pre- 
diction can drive synaptic modification and extend these conclusions from nonhuman primates to humans. The regional specificity of this modulation also suggests that information, as embodied by the relative predictability of a stimulus stream, may be a form of neural currency that can be detected with fMRI.

\section{REFERENCES}

Ashburner J, Friston KJ (1999) Nonlinear spatial normalization using basis functions. Hum Brain Mapp 7:254-266.

Berns GS, Sejnowski TJ (1998) A computational model of how the basal ganglia produce sequences. J Cognit Neurosci 10:108-121.

Bischoff-Grethe A, Proper SM, Mao K, Daniels KA, Berns GS (2000) Conscious and unconscious processing of nonverbal predictability in Wernicke's area. J Neurosci 20:1975-1981.

Bradberry CW, Barrett-Larimore RL, Jatlow P, Rubino SR (2000) Impact of self-administered cocaine and cocaine cues on extracellular dopamine in mesolimbic and sensorimotor striatum in Rhesus monkeys. J Neurosci 20:3874-3883.

Breiter HC, Gollub RL, Weisskoff RM, Kennedy DN, Makris N, Berke JD, Goodman JM, Kantor HL, Gastfriend DR, Riorden JP, Mathew RT, Rosen BR, Hyman SE (1997) Acute effects of cocaine on human brain activity and emotion. Neuron 19:591-611.

Carelli RM, Ijames SG, Crumlin AJ (2000) Evidence that separate neural circuits in the nucleus accumbens encode cocaine versus "natural" (water and food) reward. J Neurosci 20:4255-4266.

Cohen JD, Servan-Schreiber D (1992) Context, cortex, and dopamine: a connectionist approach to behavior and biology in schizophrenia. Psychol Rev 99:45-77.

Cooper JR, Bloom FE, Roth RH (1996) The biochemical basis of neuropharmacology, Ed 7. New York: Oxford UP.

Corfield DR, Murphy K, Josephs O, Fink GR, Frackowiak RSJ, Guz A, Adams L, Turner R (1999) Cortical and subcortical control of tongue movement in humans: a functional neuroimaging study using fMRI. J Appl Physiol 86:1468-1477.

Delgado MR, Nystrom LE, Fissel C, Noll DC, Fiez JA (2000) Tracking the hemodynamic responses to reward and punishment in the striatum. J Neurophysiol 84:3072-3077.

Elliott R, Friston KJ, Dolan RJ (2000) Dissociable neural responses in human reward systems. J Neurosci 20:6159-6165.

Francis S, Rolls ET, Bowtell R, McGlone F, O'Doherty J, Browning A, Clare S, Smith E (1999) The representation of pleasant touch in the brain and its relationship with taste and olfactory areas. NeuroReport 10:453-459.

Friston KJ, Frith CD, Turner R, Frackowiak RSJ (1995a) Characterising dynamic brain responses with fMRI: a multivariate approach. NeuroImage 2:166-172.

Friston KJ, Holmes AP, Worsley K, Poline JB, Frith CD, Frackowiak RSJ (1995b) Statistical parametric maps in functional brain imaging: a general linear approach. Hum Brain Mapp 2:189-210.

Friston KJ, Holmes AP, Worsely KJ (1999) How many subjects constitute a study? NeuroImage 10:1-5.

Garris PA, Kilpatrick M, Bunin MA, Michael D, Walker QD, Wightman RM (1999) Dissociation of dopamine release in the nucleus accumbens from intracranial self-stimulation. Nature 398:67-69.

Hamdy S, Mikulis DJ, Crawley A, Xue S, Lau H, Henry S, Diamant NE (1999) Cortical activation during human volitonal swallowing: an event-related fMRI study. Am J Physiol 277:G219-G225.

Houk JC, Adams JL, Barto AG (1995) A model of how the basal ganglia generate and use neural signals that predict reinforcement. In: Models of information processing in the basal ganglia (Houk JC, Davis JL, Beiser DG, eds), pp 249-270. Cambridge, MA: MIT.

Hull CL (1943) Principles of behavior. An introduction to behavior theory. New York: Appleton-Century-Crofts.

Knutson B, Westdorp A, Kaiser E, Hommer D (2000) FMRI visualization of brain activity during a monetary incentive delay task. NeuroImage 12:20-27.

Koepp MJ, Gunn RN, Lawrence AD, Cunningham VJ, Dagher A, Jones
T, Brooks DJ, Bench CJ, Grasby PM (1998) Evidence for striatal dopamine release during a video game. Nature 393:266-268.

Koob GF (1992) Drugs of abuse: anatomy, pharmacology and function of reward pathways. Trends Pharmacol Sci 13:177-184.

Krimer LS, Muly EC, Williams GV, Goldman-Rakic PS (1998) Dopaminergic regulation of cerebral microcirculation. Nat Neurosci $1: 286-289$.

Kwong KK, Belliveau JW, Chesler DA, Goldberg IE, Weisskoff RM, Poncelet BP, Kennedy DN, Hoppel BE, Cohen MS, Turner R, Cheng HM, Brady TJ, Rosen BR (1992) Dynamic magnetic resonance imaging of human brain activity during primary sensory stimulation. Proc Natl Acad Sci USA 89:5675-5679.

Mirenowicz J, Schultz W (1996) Preferential activation of midbrain dopamine neurons by appetitive rather than aversive stimuli. Nature 379:449-451

Montague PR, Dayan P, Person C, Sejnowski TJ (1995) Bee foraging in an uncertain environment using predictive Hebbian learning. Nature 376:725-728

Montague PR, Dayan P, Sejnowski TJ (1996) A framework for mesencephalic dopamine systems based on predictive Hebbian learning. J Neurosci 16:1936-1947.

Nobre AC, Coull JT, Frith CD, Mesulam MM (1999) Orbitofrontal cortex is activated during breaches of expectation in tasks of visual attention. Nat Neurosci 2:11-12.

Ogawa S, Tank DW, Menon R, Ellerman JM, Kim SG, Merkle H, Ugurbil K (1992) Intrinsic signal changes accompanying sensory stimulation: functional brain mapping with magnetic resonance imaging. Proc Natl Acad Sci USA 89:5951-5955.

Ojemann JG, Akbudak E, Snyder AZ, McKinstry RC, Raichle ME, Conturo TE (1997) Anatomic localization and quantitative analysis of gradient refocused echo-planar fMRI susceptibility artifacts. NeuroImage $6: 156-167$.

Rescorla RA, Wagner AR (1972) A theory of Pavlovian conditioning and the effectiveness of reinforcement and non-reinforcement. In: Classical conditioning. 2. Current research and theory (Black $\mathrm{AH}$, Prokasy WF, eds), pp 64-69. New York: Appleton-Century-Crofts.

Robbins TW, Everitt BJ (1996) Neurobehavioral mechanisms of reward and motivation. Curr Opin Neurobiol 6:228-236.

Robinson TE, Berridge KC (1993) The neural basis of drug craving: an incentive-sensitization theory of addiction. Brain Res Rev 18:247-291.

Rogers RD, Owen AM, Middleton HC, Williams EJ, Pickard JD, Sahakian BJ, Robbins TW (1999) Choosing between small, likely rewards and large, unlikely rewards activates inferior and orbital prefrontal cortex. J Neurosci 19:9029-9038.

Rolls ET (2000) The orbitofrontal cortex and reward. Cereb Cortex 10:284-294.

Schultz W (1998) Predictive reward signal of dopamine neurons. J Neurophysiol 80:1-27.

Schultz W, Apicella P, Scarnati E, Ljungberg T (1992) Neuronal activity in monkey ventral striatum related to the expectation of reward. J Neurosci 12:4595-4610.

Schultz W, Apicella P, Ljungberg T (1993) Responses of monkey dopamine neurons to reward and conditioned stimuli during successive steps of learning a delayed response task. J Neurosci 13:900-913.

Schultz W, Dayan P, Montague PR (1997) A neural substrate of prediction and reward. Science 275:1593-1599.

Shidara M, Aigner TG, Richmond BJ (1998) Neuronal signals in the monkey ventral striatum related to progress through a predictable series of trials. J Neurosci 18:2613-2625.

Sutton RS (1988) Learning to predict by the methods of temporal differences. Mach Learn 3:9-44.

Talairach J, Tournoux P (1988) Co-planar stereotaxic atlas of the human brain. Stuttgart, Germany: Thieme.

Tesauro G, Sejnowski TJ (1989) A parallel network that learns to play backgammon. Artif Intell J 39:357-390.

Thut G, Schultz W, Roelcke U, Nienhusmeier M, Missimer J, Maguire RP, Leenders KL (1997) Activation of the human brain by monetary reward. NeuroReport 8:1225-1228.

Tremblay L, Schultz W (1999) Relative reward preference in primate orbitofrontal cortex. Nature 398:704-708. 